\title{
ON-ORBIT OPERATIONS OF A POWER SYSTEM FOR JAPAN'S VENUS EXPLORER AKATSUKI
}

\author{
Hiroyuki Toyota $^{(1)}$, Nobuaki Ishii (1), Masato Nakamura ${ }^{(1)}$, Hiroki Ooto ${ }^{(2)}$, Kazuya Koide ${ }^{(2)}$, Tomohiko \\ Sakamoto $^{(2)}$, Hidetoshi Abe ${ }^{(2)}$, Hideki Yoshino ${ }^{(3)}$, Hiroyuki Takamura ${ }^{(3)}$, Takayuki Ose ${ }^{(3)}$, Yukishige Nozaki ${ }^{(3)}$ \\ (1) ISAS/JAXA, 3-1-1 Yoshinodai, Chuo-ku, Sagamihara, Kanagawa 252-5210, Japan, Email: htoyota@isas.jaxa.jp \\ (2) The Furukawa Battery Co., Ltd., 23-6 Kuidesaku, Joban-shimofunaomachi, Iwaki, Fukushima 972-8501, Japan, \\ Email: h-ooto@furukawadenchi.co.jp \\ (3) NEC Space Technologies, Ltd., 1-10 Nissin-cho, Fuchu, Tokyo 183-8551, Japan, Email: h-yoshino@ex.jp.nec.com
}

\begin{abstract}
This paper describes on-orbit operations of a power system for Japan's Venus explorer Akatsuki. It was launched on May 20, 2010, and approached Venus on December 7, 2010, but orbit insertion failed because of a propulsion system malfunction. After the failure, the spacecraft stayed on an orbit nearer to the sun than Venus for 5 years before successfully entering an orbit around Venus in 2015. Telemetry data that show radiation degradation of the solar arrays, and here analytical results by the relative damage coefficients method are presented. The capacity loss of the batteries was successfully suppressed by lowering the state of charge and temperature. We plan to extend the mission period from 4.5 years to longer than 9 years to obtain more fruitful scientific results.
\end{abstract}

\section{INTRODUCTION}

Akatsuki is Japan's first Venus orbiter. It observes the mysterious atmospheric circulation on Venus using five cameras covering from ultraviolet to near-infrared light. The spacecraft approached Venus as planned on December 7, 2010, but orbit insertion failed because of a propulsion system malfunction. On December 7, 2015, Akatsuki approached Venus again and was successfully inserted into an elliptic orbit around Venus. After the failed first orbit insertion, the spacecraft survived on an orbit nearer to the sun than Venus for 5 years. Now it is aiming at scientific observations on an orbit around Venus for over 2000 days, despite a design lifetime of
4.5 years in total. This paper describes the on-orbit operation of the power system for Akatsuki.

\section{OVERVIEW}

Figure 1 depicts the Akatsuki spacecraft in its flight configuration. The spacecraft mass is $502 \mathrm{~kg}$, including $189 \mathrm{~kg}$ of fuel and oxidizing agent and $33 \mathrm{~kg}$ of observation cameras. The dimensions of the main bus are $1.45 \times 1.04 \times 1.4 \mathrm{~m}$. It is equipped with two solar array paddles, which are mounted on the north and the south body surfaces and rotate to track the sun.

Figure 2 shows a block diagram of the power system for the Akatsuki spacecraft. Electrical output of the solar arrays is delivered through solar array drive assemblies (SADAs) and solar array blocking diodes (SBDs) into a series-switching regulator (SSR) to be regulated to $50 \mathrm{~V}$.

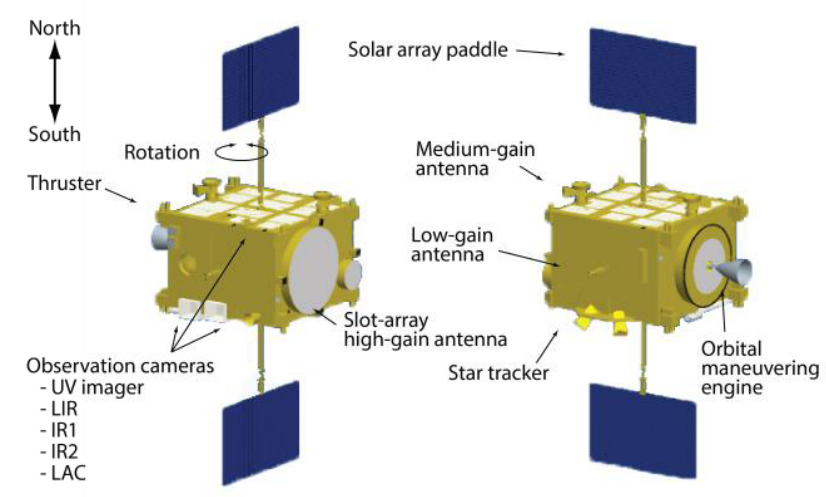

Figure 1. Akatsuki spacecraft

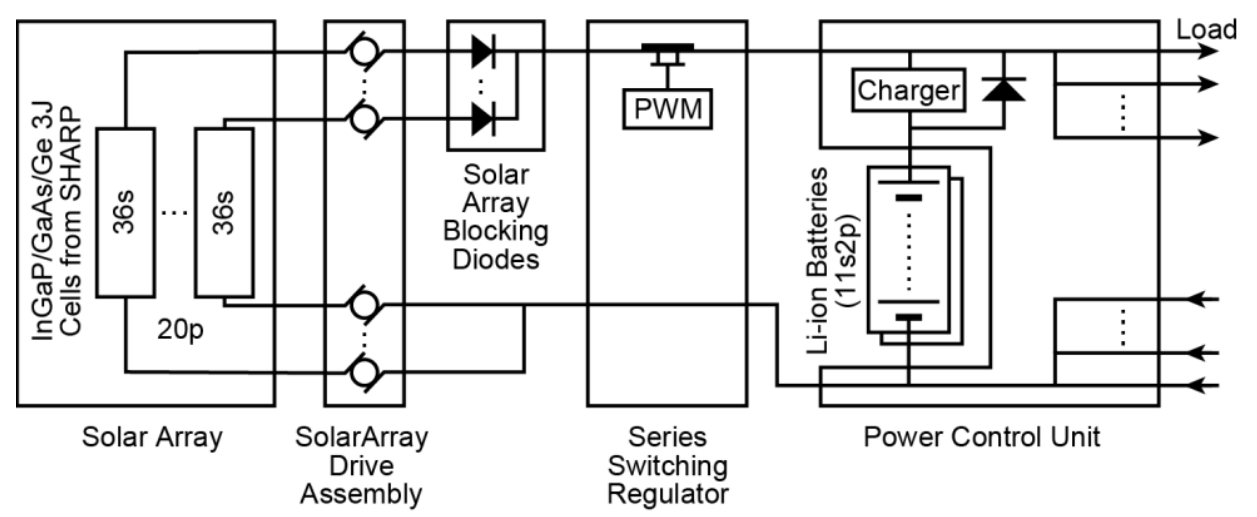

Figure 2. Block diagram of the Akatsuki power system 
A power control unit (PCU) charges and discharges lithium ion batteries, and simultaneously distributes electricity to power buses at several voltage levels.

Akatsuki was launched aboard an $\mathrm{H} 2 \mathrm{~A}$ rocket from the Tanegashima Space Center and directly injected into an orbit toward Venus on May 20, 2010. Figure 3 shows Akatsuki's trajectory. Akatsuki approached Venus on December 7, 2010 after a half year of cruise, and started burning its 500-N-class orbital maneuvering engine (OME) during Venus orbit insertion (VOI). The planned burn period was for $715.5 \mathrm{~s}$, but the OME stopped burning at $158 \mathrm{~s}$ because of a malfunction in its fuel supply line. The OME subsequently lost its nozzle, rendering it unusable. The spacecraft thus failed to enter Venus orbit, instead entering an orbit around the sun with a period of 203 days, a perihelion distance of 0.6 $\mathrm{AU}$, and an aphelion distance of $0.73 \mathrm{AU}$. After orbiting the sun nine times over five years, Akatsuki again approached Venus and conducted VOI-R1 by burning its four 23-N-class reaction control system (RCS) thrusters for $1228 \mathrm{~s}$ on December 7, 2015. The spacecraft successfully entered an elliptic orbit around Venus with a period of 13.6 days, an inclination of $3^{\circ}$, and an apoapsis altitude of $440,000 \mathrm{~km}$. To decrease the apoapsis altitude and avoid long eclipses, a trim maneuver was performed at the first periapsis, resulting in an apoapsis altitude of $360,000 \mathrm{~km}$ and a period of 10.5 days. The orbit will be continuously adjusted to extend the mission life from the originally planned 800 days to 2000 days on Venus orbit.

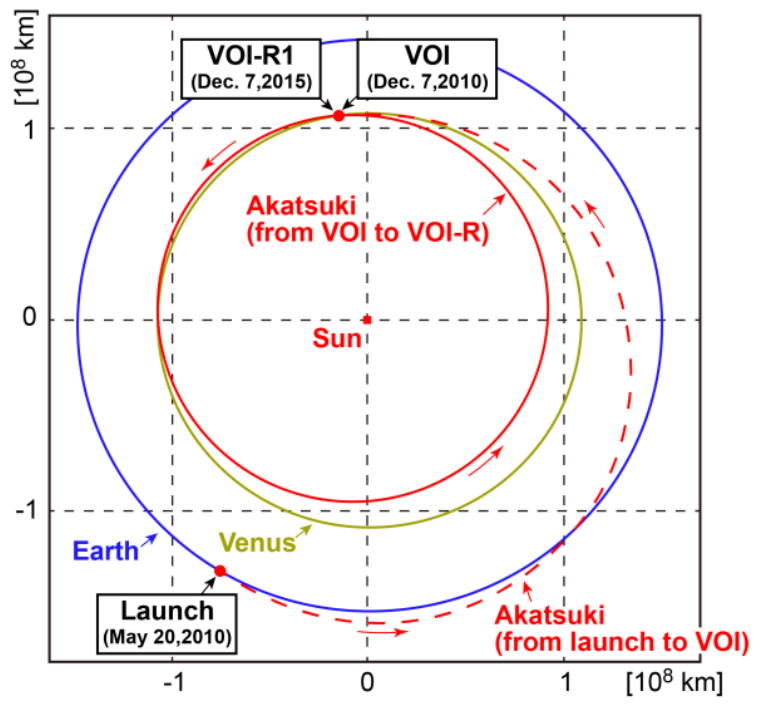

Figure 3. Trajectory of Akatsuki from launch to Venus orbit insertions VOI and VOI-RI.

\section{SOLAR ARRAY PANELS}

Figure 4 shows a solar array panel of ESA's Venus Express, which was operated until about a year before Akatsuki entered an orbit around Venus. On the solar array panels of Venus Express, solar cell strings and optical solar reflector strings are mounted alternately to lower the panel temperature.

Akatsuki, on the other hand, was originally planned to be launched aboard a small solid rocket, M-V, which was far smaller than the $\mathrm{H} 2 \mathrm{~A}$ rocket. The solar array panels of Akatsuki were designed to fit in the $\mathrm{M}-\mathrm{V}$ payload fairing, which has a diameter of $1.8 \mathrm{~m}$. As a result, the front side of Akatsuki's solar array panel, which was $1.43 \mathrm{~m}$ wide and $1.036 \mathrm{~m}$ long, was covered by $\mathrm{InGaP} / \mathrm{GaAs} / \mathrm{Ge} 3 \mathrm{~J}$ solar cells from Sharp Corp. to obtain the required electrical power, as shown in Figure 5 . The solar cells are covered by $100-\mu$ m-thick CMG cover glasses with AR coats. The predicted generated power is more than $480 \mathrm{~W}$ at $1.0781 \mathrm{AU}$ and more than $660 \mathrm{~W}$ on the Venus orbit. The rear side was covered by optical solar reflectors (OSRs) to lower albedo input from Venus. The designed temperature range of the solar array panels was from $-170{ }^{\circ} \mathrm{C}$ to $+184^{\circ} \mathrm{C}[3]$.

Figure 6 shows telemetry data of the solar array output voltage and temperature. Between VOI and VOI-R1, the solar array temperature varied from $+100{ }^{\circ} \mathrm{C}$ to $+140{ }^{\circ} \mathrm{C}$, depending on the distance to the sun. The output voltage varied according to the temperature, and gradually decreased over time due to radiation damage caused by solar protons. The output voltage trend also shows four sudden drops, which are assumed to have been caused by solar flares [4].

We analyzed radiation damage to the solar array by the relative damage coefficients (RDCs) method [5]. Figure 7 shows the RDCs of the solar cells used in the analysis. Figure 8 shows the constant solar proton environment that caused the gradual voltage drop estimated by the SPENVIS [6].

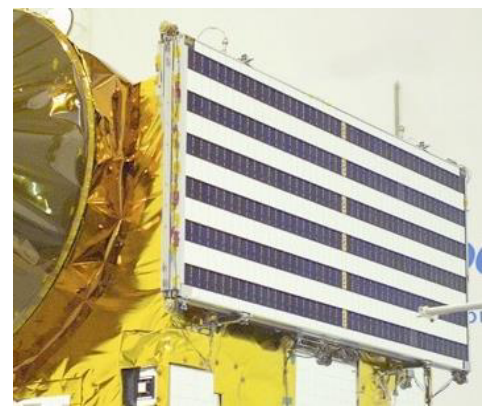

Figure 4. Solar array panel on Venus Express [2]

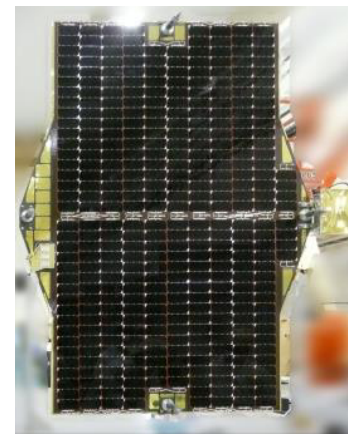

(a) Front side

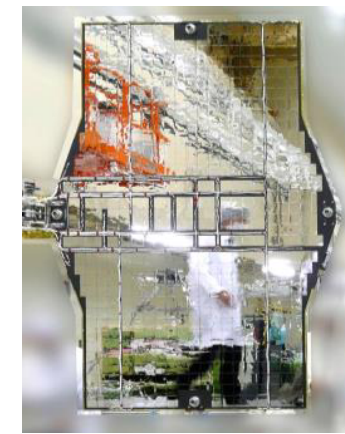

(b) Rear side
Figure 5. Solar array panels on Akatsuki 


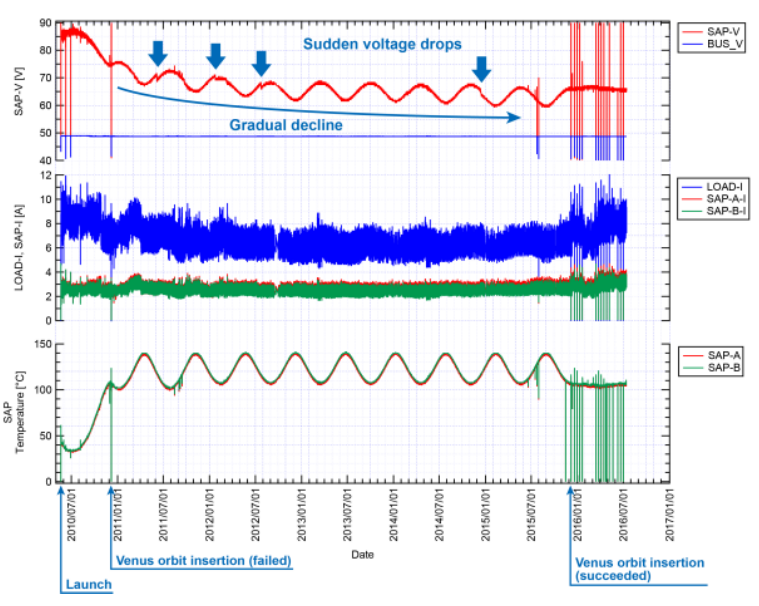

Figure 6. Telemetry data of solar array output voltage and temperature

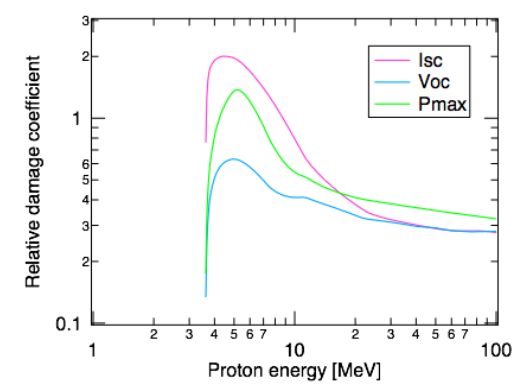

Figure 7. Relative damage coefficients of solar cells used in analysis

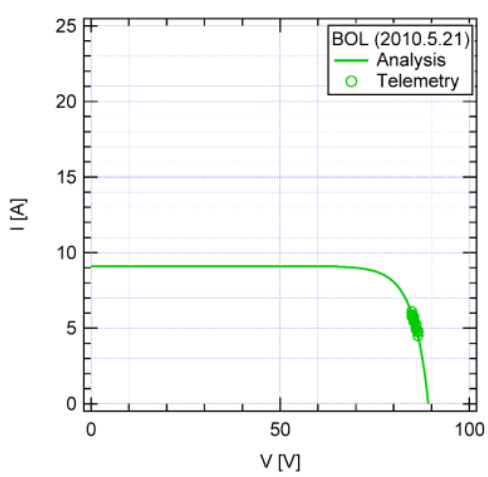

(a) BOL (May 20, 2010)

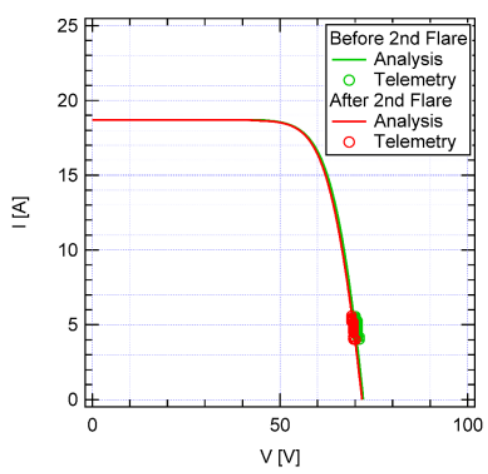

(d) 2nd flare (Jan. 23, 2012)

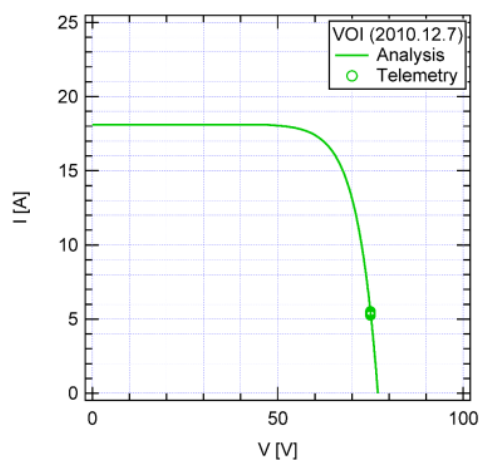

(b) $\operatorname{VOI}($ Dec. 7,2010$)$

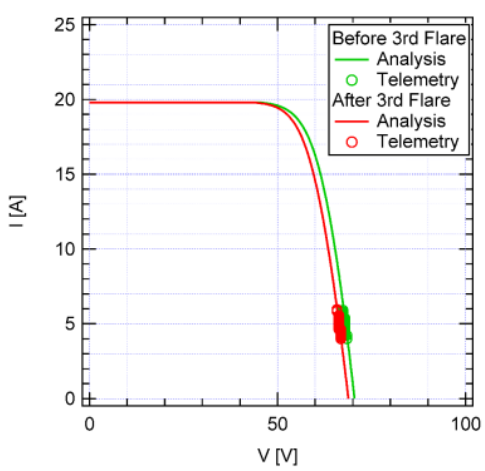

(e) 3rd flare (July 23, 2012)

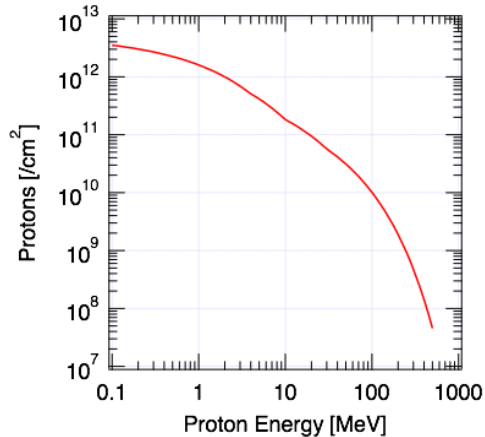

(a) Solar proton spectrum accumulated from May 21, 2010 to December 7, 2017

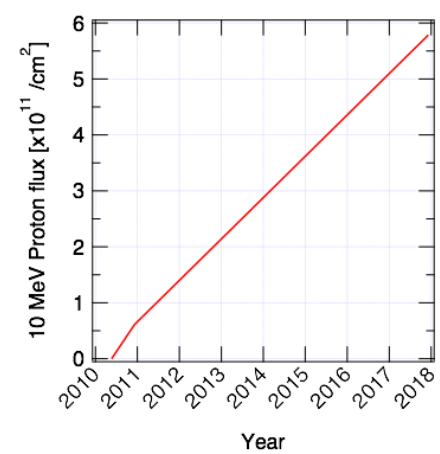

(b) Time evolution of equivalent 10-MeV proton fluence for $P_{\max }$

Figure 8. Solar proton environment

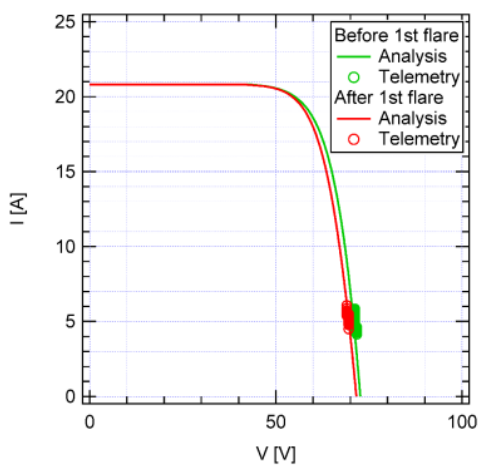

(c) 1st flare (June 5, 2011)

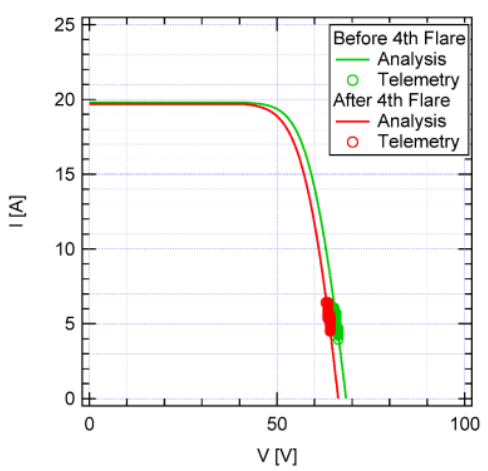

(f) 4th flare (Dec. 13, 2014)

Figure 9. Current-voltage characteristics of the solar array as calculated by the RDC method and telemetry data 
For each sudden voltage drop event, we estimated a proton dose that matches the telemetry data, because there are no observation data available.

Figure 9 shows the analytical results and telemetry data. The calculated current-voltage (IV) characteristics by the RDC method agree well with the telemetry data when the radiation damage margin (RDM) is 0.5 . This is plausible, because the solar activity was lower than expected over this period [7].

Figure 10 shows IV and power-voltage (PV) characteristics at BOL, VOI, VOI-R1, and 2000 days from VOI-R1 calculated using the RDC method. The power required by Akatsuki, which is less than $500 \mathrm{~W}$ at $56 \mathrm{~V}$, can be generated even 2000 days from VOI-R1.

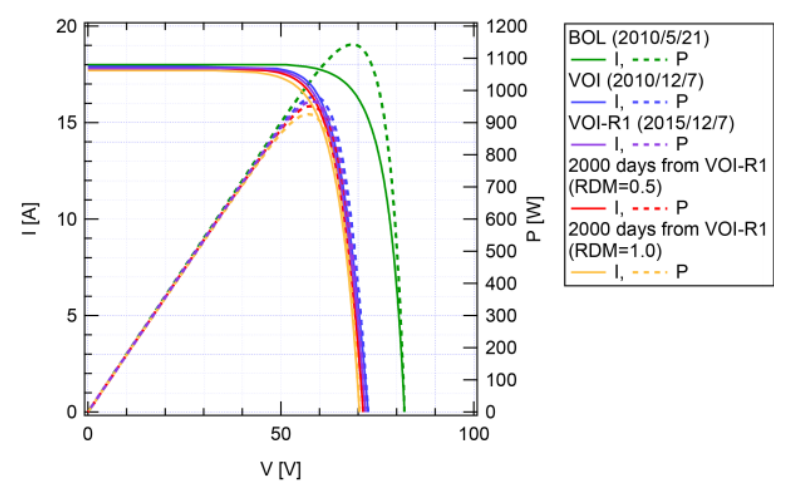

Figure 10. Current-voltage and power-voltage characteristics calculated using RDCs

\section{BATTERIES}

Akatsuki carries two 23.5-Ah lithium-ion batteries connected in parallel. Each battery consists of eleven cells connected in series and protection circuits (Figure 11). The capacity of lithium ion batteries degrades faster at higher temperatures and at a higher state of charge (SOC). We therefore planned to keep the temperature and the SOC as low as possible to minimize capacity degradation.

Figure 12 and Table 1 show the original battery operation plan. When not in use in ground tests, the batteries were kept at $10 \%$ SOC in open circuit at room temperature for 1.5 years prior to launch to prevent over-discharge. After the spacecraft was launched, the batteries were kept at $41 \% \mathrm{SOC}$ at $10{ }^{\circ} \mathrm{C}$ in preparation for recovery from an unexpected attitude loss of the spacecraft until VOI (except during reset operations, in which all cells were charged to $4.1 \mathrm{~V}$ then discharged to balance cell voltages). This period was 0.5 years, while the longest trajectory was supposed to take 2.5 years. After VOI, the batteries were used as a power supply during eclipses for two years. During this period, the batteries are repeatedly charged and discharged 550 times by changing the SOC from $41 \%$ to $85 \%$ depending on the eclipse duration, which could be up to 90 minutes. [8]

After Akatsuki failed in the VOI on December 7, 2010, we revised the operation plan as shown in Figure 13 and Table 2 to reduce the capacity loss and support an extended mission. The SOC was reduced from $41 \%$ to $30 \%$ and the temperature was lowered from $10{ }^{\circ} \mathrm{C}$ to $0{ }^{\circ} \mathrm{C}$, based on the capacity actually required when the spacecraft recovered from its attitude loss during the failed VOI.

After the successful VOI-R1, Akatsuki entered an elliptic orbit around Venus with an apoapsis altitude

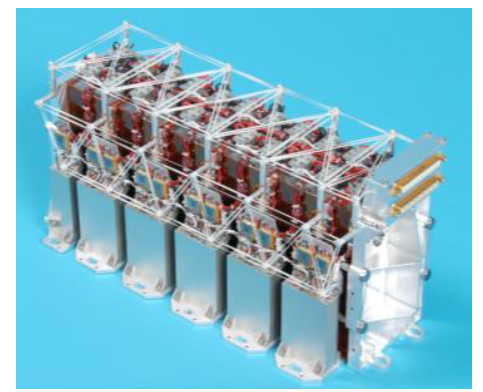

Figure 11. Lithium ion battery for Akatsuki

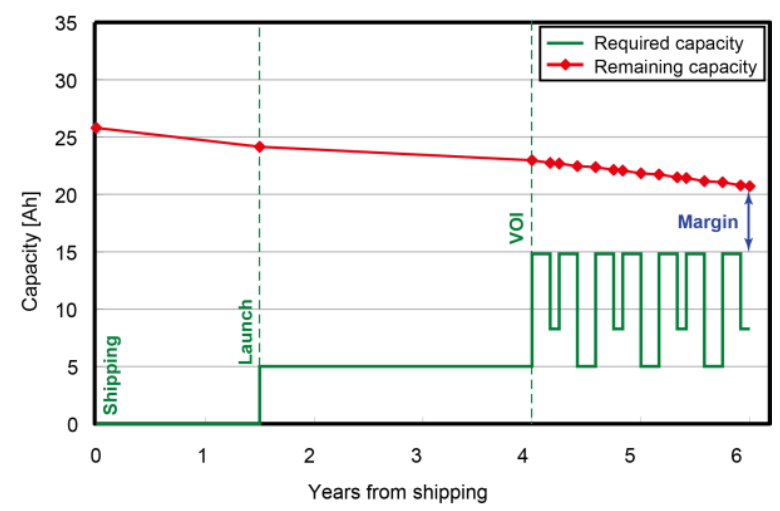

Figure 12. Originally planned required capacity and predicted trend of battery capacity

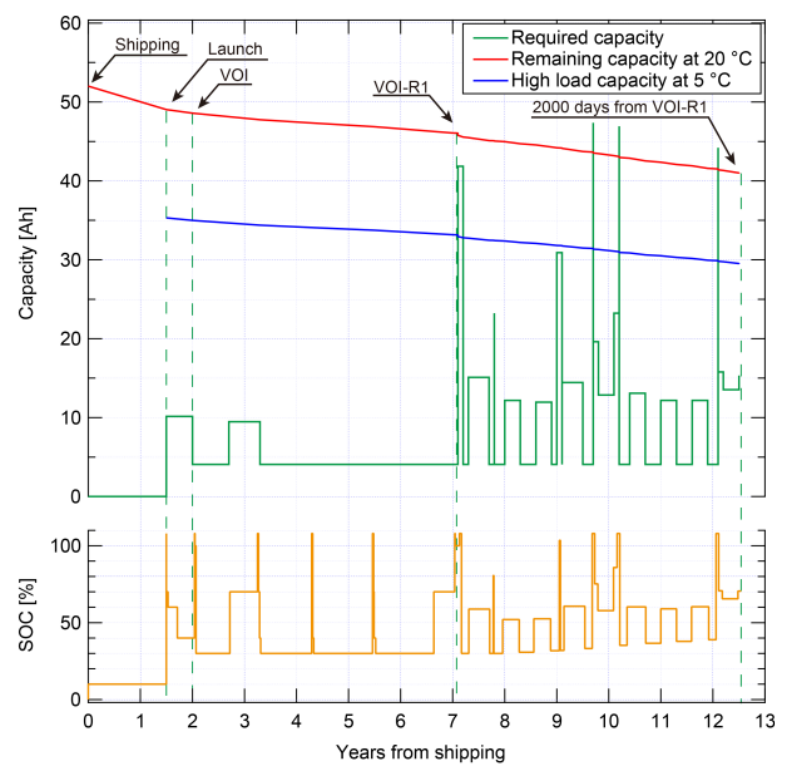

Figure 13. Required capacity and predicted trend of battery capacity revised after VOI and VOI-RI 
Table 1. Original battery operation plan

\begin{tabular}{|c|c|c|c|c|c|}
\hline \multicolumn{2}{|c|}{ Mission phase } & Time [Years] & $\mathrm{SOC}[\%]$ & Temperature $\left[{ }^{\circ} \mathrm{C}\right]$ & Battery state \\
\hline \multicolumn{2}{|c|}{ Shipping to launch } & 1.5 & 10 & 25 & Open circuit \\
\hline \multirow{2}{*}{$\begin{array}{l}\text { Launch to } \\
\text { VOI }\end{array}$} & Nominal & 0.5 & \multirow{2}{*}{41} & \multirow{2}{*}{10} & \multirow{2}{*}{ Float charge } \\
\hline & Backup & 2.5 & & & \\
\hline \multicolumn{2}{|c|}{ Venus orbit } & 2 & $41-85$ & 10 & Charge and discharge cycle \\
\hline
\end{tabular}

Table 2. Battery operation plan revised after failed VOI

\begin{tabular}{|c|c|c|c|c|}
\hline Mission phase & Time [Years] & SOC $[\%]$ & Temperature $\left[{ }^{\circ} \mathrm{C}\right]$ & Battery state \\
\hline Shipping to launch & 1.5 & 10 & 25 & Open circuit \\
\hline Launch to VOI & 0.5 & 41 & 10 & \multirow{2}{*}{ Float charge } \\
\hline VOI to VOI-R1 & 5 & 30 & 0 & Charge and discharge cycle \\
\hline Venus orbit & 6 & $30-108$ & $5-20$ & \\
\hline
\end{tabular}

much greater than originally planned. As a result, the longest eclipse duration was extended from 90 minutes to 7.9 hours and the greatest required energy during an eclipse increased from $750 \mathrm{Wh}$ to $1600 \mathrm{Wh}$, while the number of the eclipses was reduced from 550 times to 110 times over 2000 days on Venus orbit. As shown in Figure 13, eclipses at 9.7, 10.2, and 12.1 years after shipping require capacities larger than the remaining capacities. During these eclipses, the batteries will meet the requirements by being charged to $108 \%$ SOC and putting the spacecraft into survival mode, which requires less power than nominal mode.

\section{SUMMARY}

This paper described the operation of Japan's Venus explorer Akatsuki. The spacecraft's orbit insertion failed because of a malfunction in its OME, but successfully entered an orbit around Venus using its thrusters for attitude control after orbiting around the sun for 5 years, despite a designed spacecraft life of 4.5 years. Radiation degradation of the solar array was analyzed by the RDC method, and the results agreed well with telemetry data. The battery life was extended by lowering the SOC and the temperature to meet the demands of a mission extended to 12.5 years. Akatsuki has already smoothly started scientific observations and a mission life of 2000 days on Venus orbit is expected.

\section{REFERENCES}

1. Masato Nakamura et al. (2016). AKATSUKI return to Venus. Earth, Planets and Space. 68:75.

2. http:/www.esa.int/spaceinimages/Images/2005/08/ Venus_Express_ready_for_shipping_to_Baikonur2

3. Hiroyuki Toyota et al. (2008). Venus Climate Orbiter Planet-C Solar Array Development, in Proc. 8th European Space Power Conference, European Space Agency, Constantz, Germany.

4. Yu Takahashi et al. (2014). Analysis of Radiation Damage in On-orbit Solar Array of Venus Explorer Akatsuki, in Pro. $9^{\text {th }}$ European Space Power Conference, European Space Agency, Noordwijkerhout, The Netherlands.
5. B. E. Anspaugh (1996). GaAs Solar Cell Radiation Handbook, Jet Propulsion Laboratory Publication, 96-9.

6. SPENVIS web site. https://www.spenvis.oma.be/

7. Ian G. Richardson et al. (2016). $25 \mathrm{MeV}$ Solar Proton Events in Cycle 24 and Previous Cycles, Advances in Space Research.

8. Hiroki Ooto et al. (2008). Development of Lithium-Ion Battery for the Venus Climate Orbiter "PLANET-C", in Proc. 8th European Space Power Conference, European Space Agency, Constantz, Germany. 